\title{
Modèle de contrat de travail pour assistantes médicales et nouvelle allocation de maternité
}

\author{
Dr Michel Marchev, Président des délégués aux questions des assistantes médicales
}

Dans le cadre de l'allocation de maternité entrant en vigueur le $1^{\text {er }}$ juillet 2005 , le contrat de travail type des assistantes médicales a été adapté. Vous trouvez la nouvelle version sur la page internet de la FMH $\rightarrow$ www.fmh.ch $\rightarrow$ Nos prestations $\rightarrow$ assistantes médicales $\rightarrow$ documents-types $\rightarrow$ modèles de contrat. Il faut relever qu'aucun nouveau contrat ne doit être conclu, les nouvelles dispositions ayant la priorité sur les conventions de droit privé. Les conventions déjà signées continuent d'être valables.

Il convient de noter que la nouvelle allocation n'est valable qu'à partir de la naissance. L'incapacité de travail avant l'accouchement est remboursée selon les dispositions du chiffre 8.1 (rétribution en cas d'empêchement de travailler). Les nécessaires adaptations du contrat-type de travail pour les nouveaux engagements ont été conçues avec l'accord des délégués cantonaux aux questions des assistantes médicales, en conservant la perspective favorable à l'employée adoptée jusqu'ici. Les prestations de l'employeur sont donc plus élevées que le minimum légal.

Je rappelle l'article informatif de Mme Lucia Rabia, juriste à la FMH, paru dans le BMS [1]. Il répond à la plupart des questions, notamment en ce qui concerne les dispositions transitoires.

\section{Complément d'information sur le plan juridique}

Lucia Rabia, service juridique de la FMH

Mon dernier article sur la nouvelle allocation de maternité [1] ne traitait pas la question suivante: dans quelle mesure le chiffre 8.3 de l'ancien contrat-type pour assistantes médicales est-il en- core applicable en cas de rapport de travail non interrompu, si l'employée met un enfant au monde après le $1^{\text {er }}$ juillet 2005 ?

Les nouvelles dispositions légales ne mentionnent rien à ce propos, mais elles rendent caducs de par la loi les contrats d'assurance prévoyant des indemnités journalières en cas de maternité. Les contrats de travail individuels dotés de dispositions favorables aux salariées demeurent en l'état, car c'était la volonté des parties contractantes que l'employée dispose d'un meilleur statut que celui prévu par la loi.

Si vous avez signé l'ancien contrat-type pour assistantes médicales, vous avez l'obligation, en tant qu'employeur, de continuer à verser le salaire complet pendant 4 semaines déjà au cours de la première année de service et pendant 8 semaines à partir de la deuxième année de service. Durant cette période, vous bénéficierez cependant des allocations pour perte de gain (APG) et ne devrez, à l'avenir, plus verser la part de prime pour l'assurance d'indemnités journalières en cas de maternité éventuellement conclue avant ce délai.

Comme mentionné précédemment, il n'est pas nécessaire de conclure un nouveau contrat de travail. L'employeur et son employée ont néanmoins la possibilité de le faire d'un commun accord. Si le rapport de travail est reconduit avec le contrat existant, l'obligation de verser le salaire précitée s'applique encore durant plusieurs années.

Référence

1 Rabia L. L'allocation de maternité entre en vigueur le $1^{\text {er }}$ juillet 2005. Bull Méd Suisse 2005; 86(11):669-70. 